\title{
Synthesis and Characterization of New Phenylbis(salicylato)bismuth(III) Complexes
}

\author{
Vitalie Stavila $^{1}$, James C. Fettinger ${ }^{2}$ and Kenton H. Whitimire ${ }^{1 *}$ \\ ${ }^{I}$ Department of Chemistry, MS 60, Rice University, 6100, Main Street, Houston, Texas 77005-1892 \\ ${ }^{2}$ Department of Chemistry, University of California, One Shields Ave., Davis, California 95616-5298 \\ *E-mail: whitmir@ rice.edu
}

\section{SUPPORTING INFORMATION}

Figure S1. Observed and calculated isotope distributions for (a) $[\mathrm{BiPh}(\mathrm{Hsal})(\mathrm{sal})]^{-}$and (b) $\left[\mathrm{Bi}_{2} \mathrm{Ph}_{2}(\mathrm{Hsal})(\mathrm{sal})_{2}\right]^{-}$in ESI-MS.

Figure S2. Observed and calculated isotope distributions for (a) $\left[\mathrm{BiPh}\left(\mathrm{Hsal}^{4 \mathrm{Me}}\right)\left(\mathrm{sal}^{4 \mathrm{Me}}\right)\right]^{-}$and (b) $\left[\mathrm{Bi}_{2} \mathrm{Ph}_{2}\left(\mathrm{Hsal}^{4 \mathrm{Me}}\right)\left(\mathrm{sal}^{4 \mathrm{Me}}\right)_{2}\right]^{-}$in ESI-MS.

Figure S3. ${ }^{13} \mathrm{C}\left\{{ }^{1} \mathrm{H}\right\}$ NMR spectra of (a) $\mathbf{1}$ and (b) 2 in $\mathrm{d}_{6}$-dmso $(400 \mathrm{MHz})$.

Figure S4. ${ }^{1} \mathrm{H}-{ }^{1} \mathrm{H}$ COSY NMR spectra of 3 and $\mathbf{4}$ in $\mathrm{d}_{6}$-dmso (500 MHz).

Figure S5. Coordination environment of $\mathrm{Bi}(\mathrm{III})$ in the two molecules of the asymmetric unit of 4 .

Table. Selected geometric parameters for 4 .

Figure S6. Coordination environment of $\mathrm{Bi}(\mathrm{III})$ in $\left[\mathbf{9} \cdot \mathrm{Me}_{2} \mathrm{CO}\right]_{2}$ and $\left[\mathbf{1 0} \cdot 2 \mathrm{Me}_{2} \mathrm{CO}\right]_{2}$.

Figure S7. The negative-ion ESI mass spectrum of $\mathbf{1 2}$ run in an acetone mobile phase.

Figure S8. Comparison of experimental and calculated negative-ion ESI mass spectral isotope patterns for $\left[\mathrm{Bi}_{9} \mathrm{O}_{7}\left(\mathrm{Hsal}^{4 \mathrm{Me}}\right)_{13}\left(\mathrm{sal}^{4 \mathrm{Me}}\right)\right]^{2-}$ and $\left[\mathrm{Bi}_{10} \mathrm{O}_{8}\left(\mathrm{Hsal}^{4 \mathrm{Me}}\right)_{14}\left(\mathrm{sal}^{4 \mathrm{Me}}\right)\right]^{2}$.

Figure S9. Comparison of experimental and calculated negative-ion ESI mass spectral isotope patterns for $\left[\mathrm{Bi}_{10} \mathrm{O}_{8}\left(\mathrm{Hsal}^{4 \mathrm{Me}}\right)_{14}\left(\mathrm{sal}^{4 \mathrm{Me}}\right)\left(\mathrm{Me}_{2} \mathrm{CO}\right)_{2}\left(\mathrm{H}_{2} \mathrm{O}\right)_{2}\right]^{2-}$ and $\left[\mathrm{Bi}_{10} \mathrm{O}_{8}\left(\mathrm{Hsal}^{4 \mathrm{Me}^{2}}\right)_{14}\left(\mathrm{sal}^{4 \mathrm{Me}}\right)\left(\mathrm{Me}_{2} \mathrm{CO}\right)_{2}\left(\mathrm{H}_{2} \mathrm{O}\right)_{5}\right]^{2-}$. 

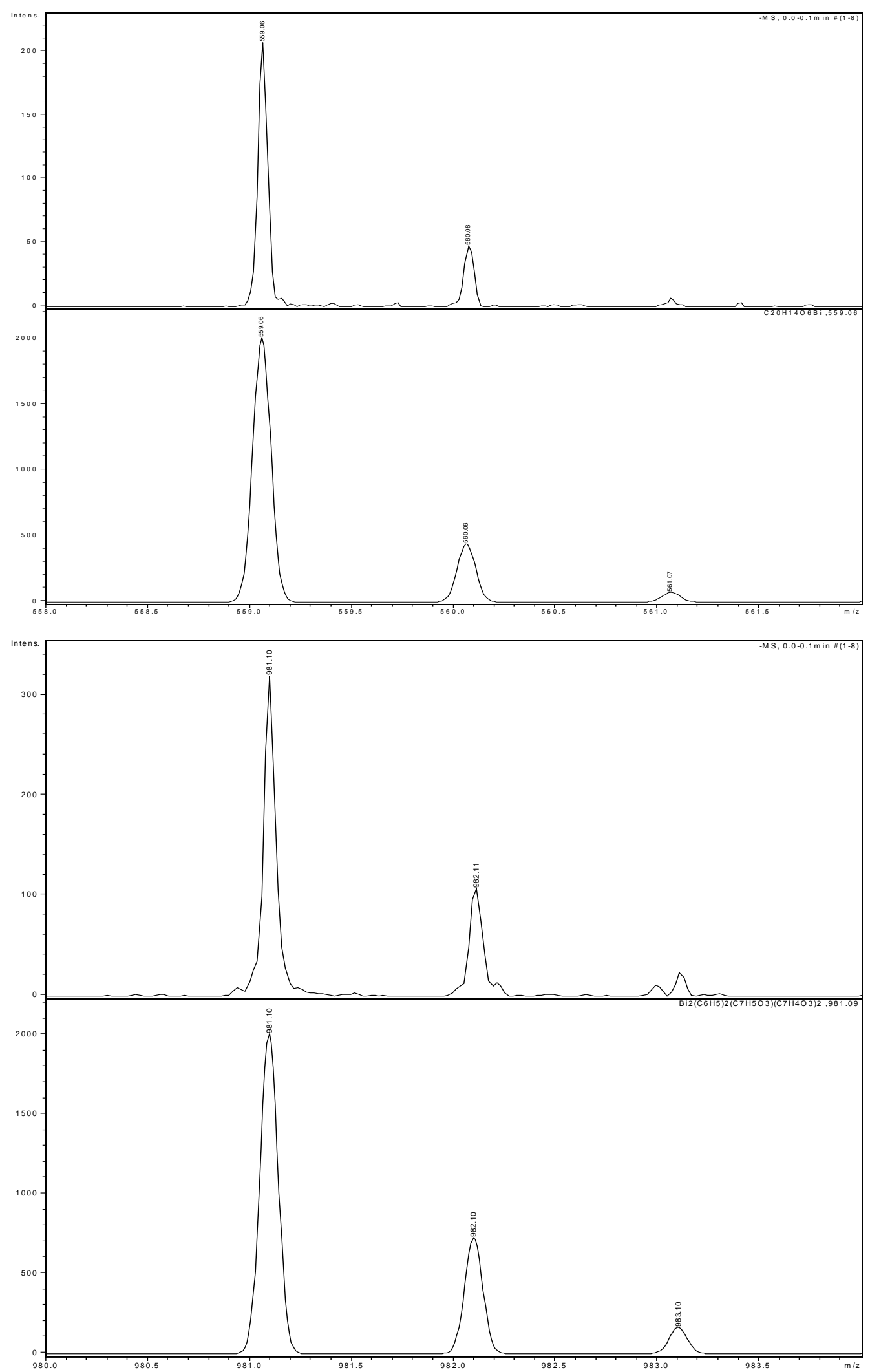

Figure S1. Observed and calculated isotope distributions for (a) $[\mathrm{BiPh}(\mathrm{Hsal})(\mathrm{sal})]^{-}$and (b) $\left[\mathrm{Bi}_{2} \mathrm{Ph}_{2}(\mathrm{Hsal})(\mathrm{sal})_{2}\right]^{-}$in ESI-MS. 

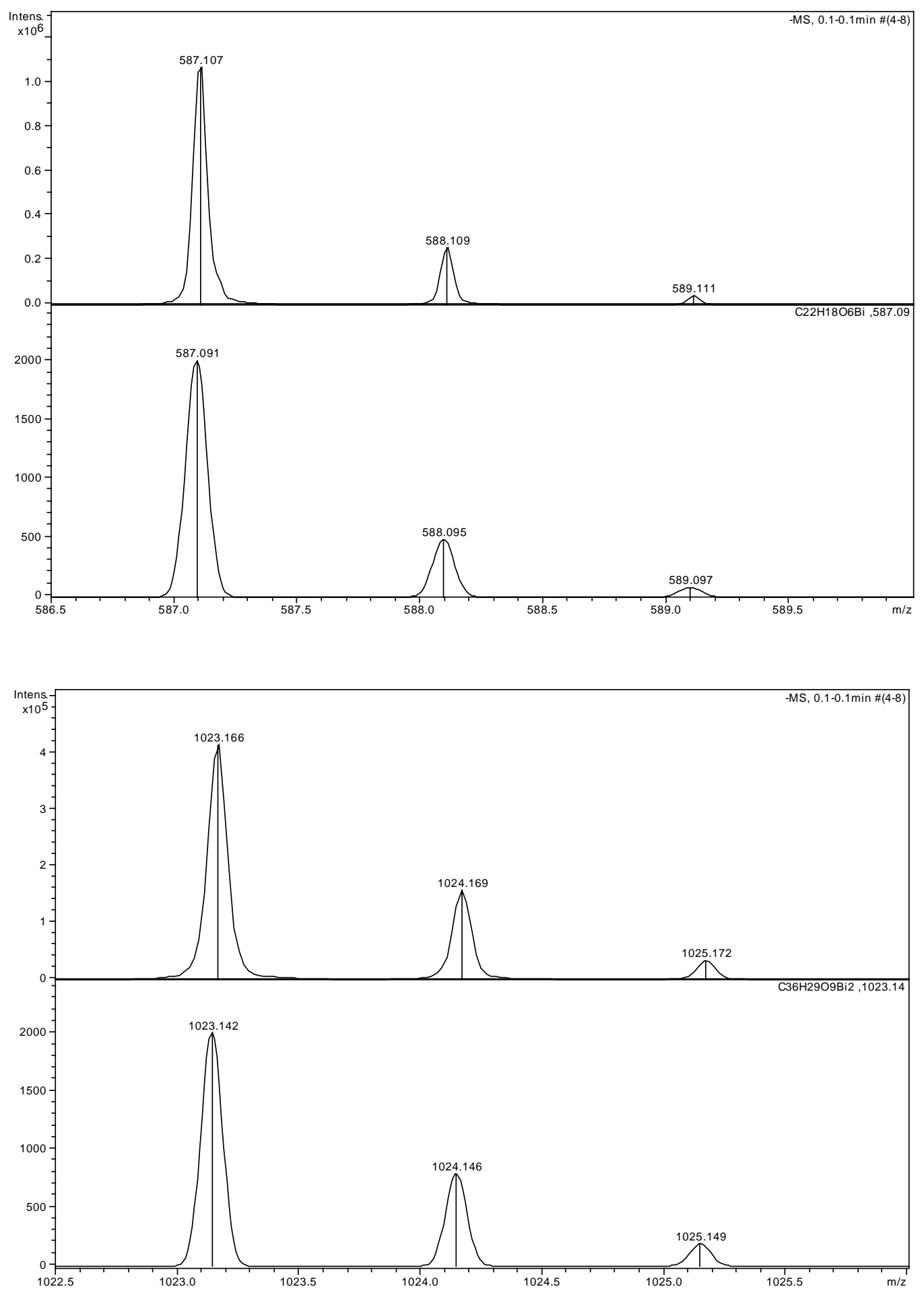

Figure S2. Observed and calculated isotope distributions for (a) $\left[\mathrm{BiPh}\left(\mathrm{Hsal}^{4 \mathrm{Me}}\right)\left(\mathrm{sal}^{4 \mathrm{Me}}\right)\right]^{-}$and (b) $\left[\mathrm{Bi}_{2} \mathrm{Ph}_{2}\left(\mathrm{Hsal}^{4 \mathrm{Me}}\right)\left(\mathrm{sal}^{4 \mathrm{Me}}\right)_{2}\right]^{-}$in ESI-MS. 
(a)
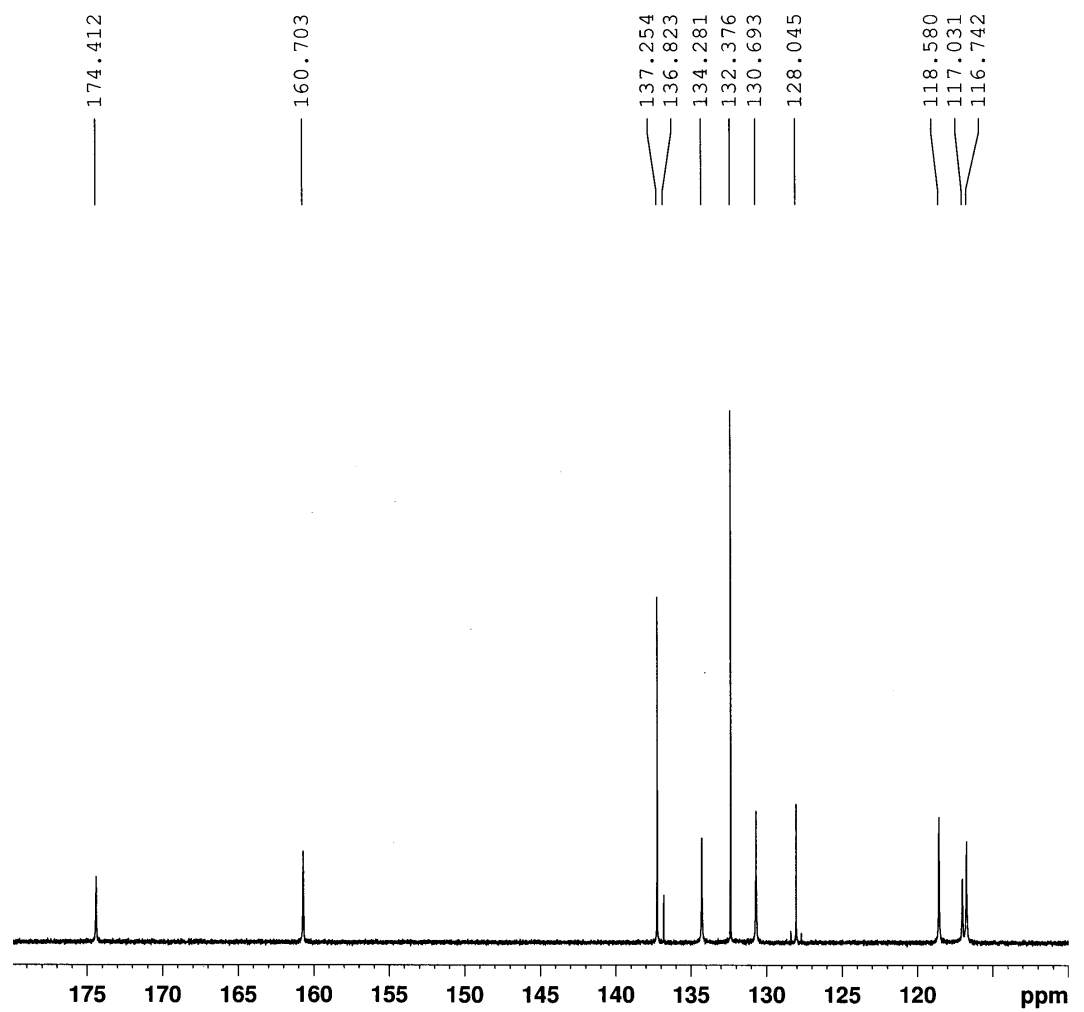

(b)

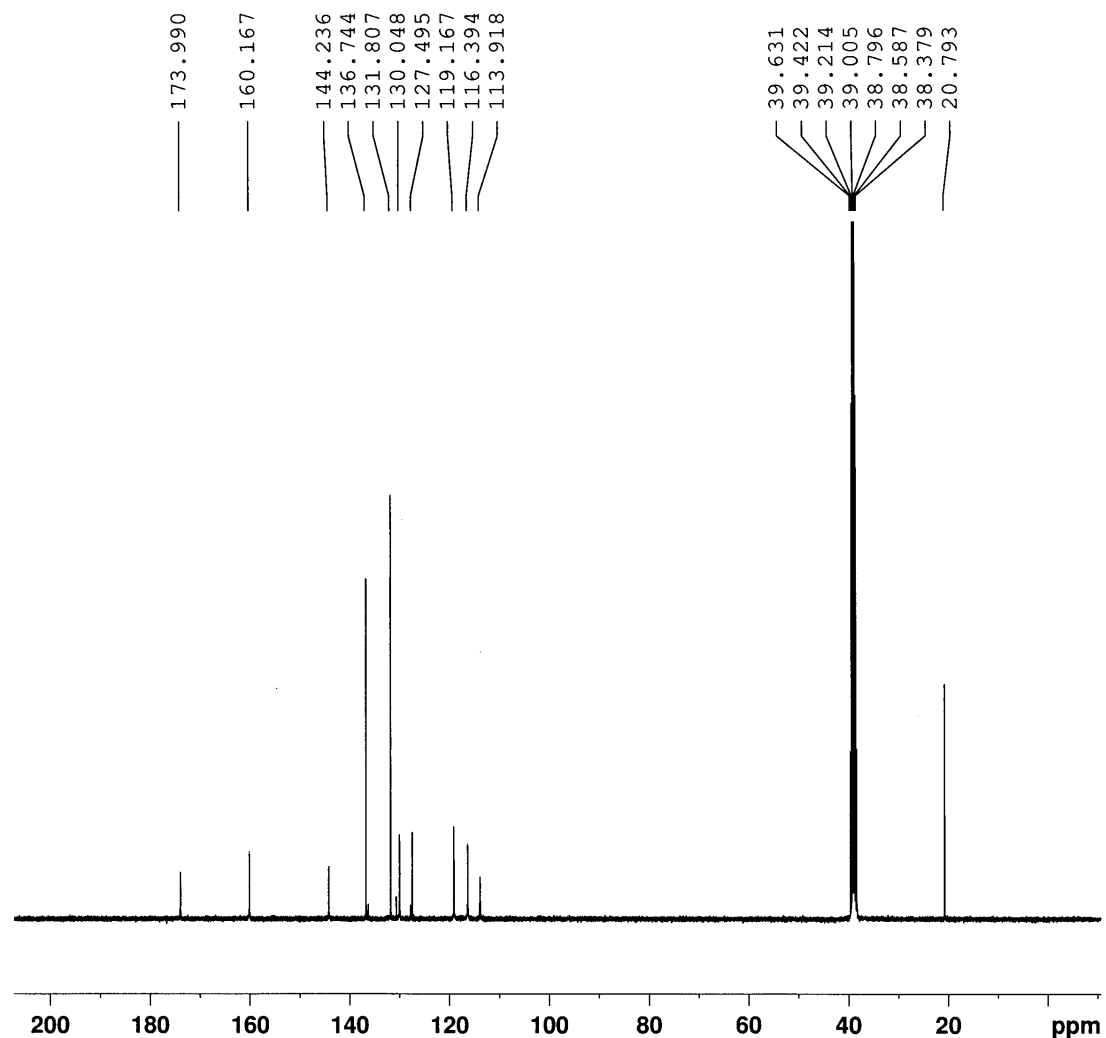

Figure S3. ${ }^{13} \mathrm{C}\left\{{ }^{1} \mathrm{H}\right\}$ spectra of (a) $\mathbf{1}$ and (b) $\mathbf{2}$ in $\mathrm{d}_{6}$-dmso (400 MHz). 
(a)

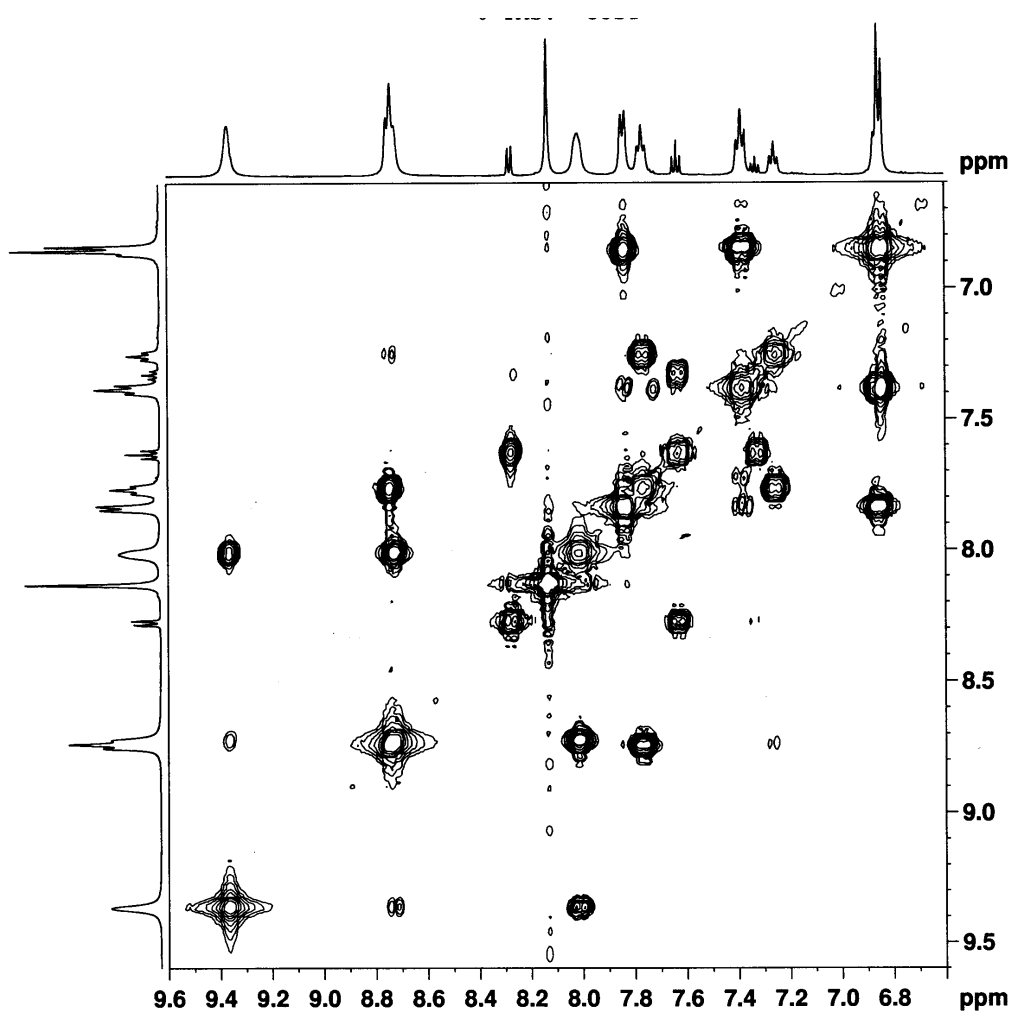

(b)

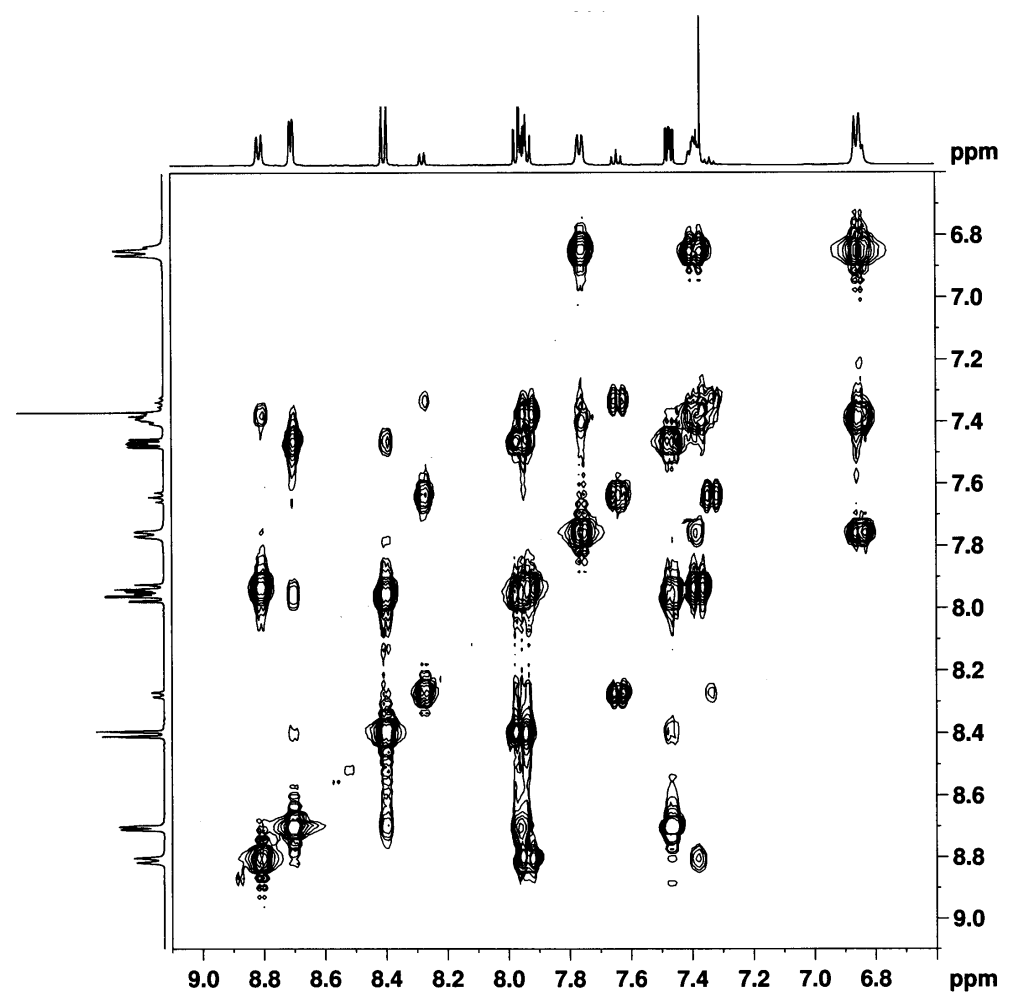

Figure S4. ${ }^{1} \mathrm{H}-{ }^{1} \mathrm{H}$ COSY NMR spectra of (a) 3 and (b) 4 in $\mathrm{d}_{6}$-dmso (500 MHz). Low intensity signals at $\sim 8.3 \mathrm{ppm}$, and the pair of triplets at $\sim 7.6 \mathrm{ppm}$ and $\sim 7.3$ may be attributed to the presence of traces of diphenylbismuth(salicylate) species in solutions. 

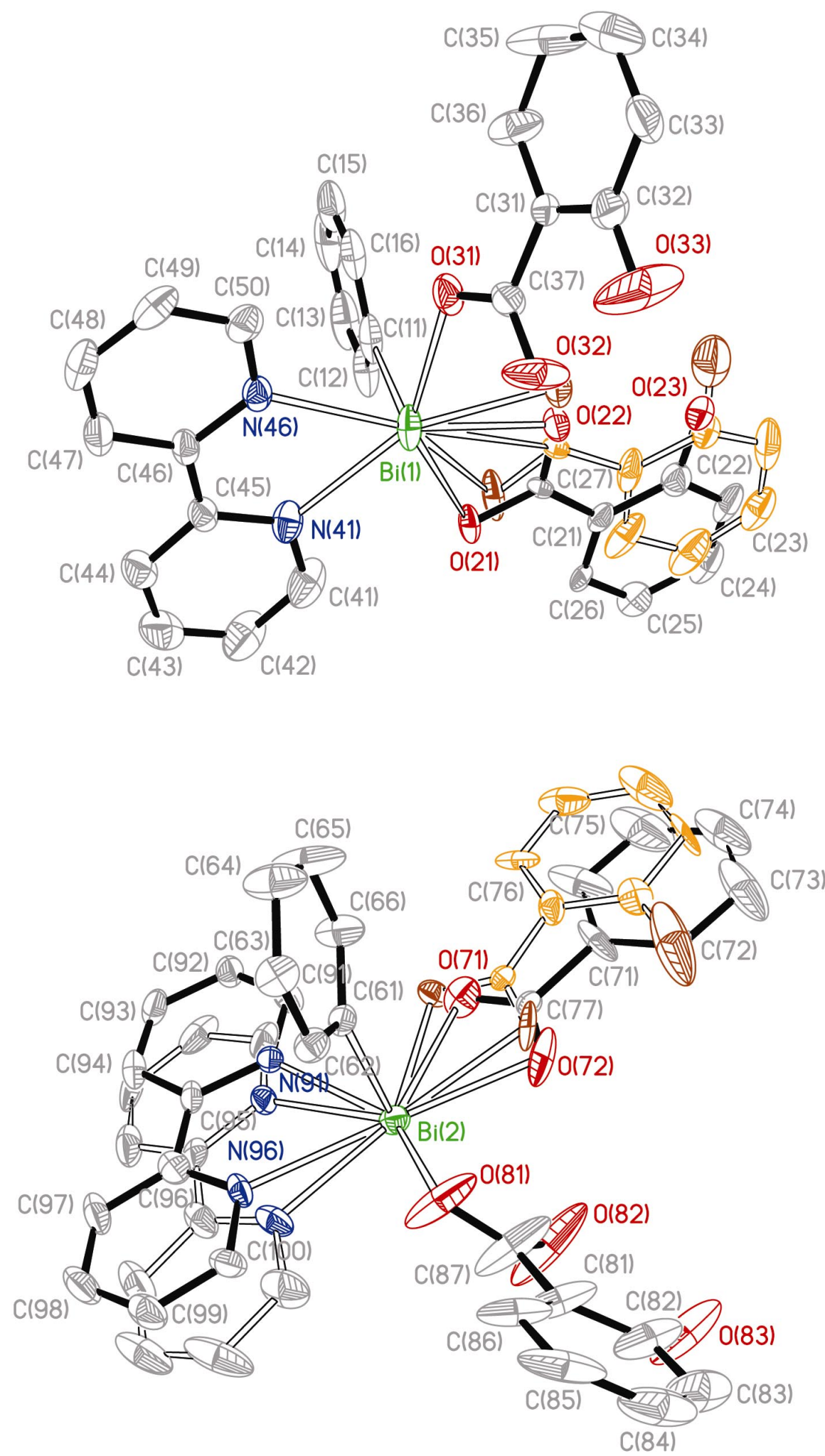

Figure S5. Coordination environment of $\mathrm{Bi}(\mathrm{III})$ in the two molecules of the asymmetric unit of 4 . 
Table. Selected geometric parameters for 4 .

\begin{tabular}{|c|c|}
\hline & Bond distances $(\AA)$ \\
\hline $\mathrm{Bi}(1)-\mathrm{C}(11)$ & $2.250(3)$ \\
\hline $\mathrm{Bi}(1)-\mathrm{O}(31)$ & $2.330(4)$ \\
\hline $\operatorname{Bi}(1)-\mathrm{N}(46)$ & $2.521(2)$ \\
\hline $\mathrm{Bi}(1)-\mathrm{O}(22)$ & $2.533(7)$ \\
\hline $\mathrm{Bi}(1)-\mathrm{O}(21)$ & $2.538(7)$ \\
\hline $\mathrm{Bi}(1)-\mathrm{O}(21 \mathrm{~A})$ & $2.524(7)$ \\
\hline $\mathrm{Bi}(1)-\mathrm{O}(22 \mathrm{~A})$ & $2.531(7)$ \\
\hline $\operatorname{Bi}(1)-N(41)$ & $2.546(3)$ \\
\hline $\mathrm{Bi}(1)-\mathrm{C}(27 \mathrm{~A})$ & $2.854(11)$ \\
\hline $\mathrm{Bi}(2)-\mathrm{C}(61)$ & $2.251(2)$ \\
\hline $\mathrm{Bi}(2)-\mathrm{O}(81)$ & $2.316(4)$ \\
\hline Bi(2)-N(96A) & $2.467(5)$ \\
\hline $\mathrm{Bi}(2)-\mathrm{N}(91 \mathrm{~A})$ & $2.483(4)$ \\
\hline $\mathrm{Bi}(2)-\mathrm{O}(72)$ & $2.491(7)$ \\
\hline $\mathrm{Bi}(2)-\mathrm{O}(71)$ & $2.495(7)$ \\
\hline $\mathrm{Bi}(2)-\mathrm{O}(71 \mathrm{~A})$ & $2.496(7)$ \\
\hline $\mathrm{Bi}(2)-\mathrm{O}(72 \mathrm{~A})$ & $2.510(7)$ \\
\hline $\mathrm{Bi}(2)-\mathrm{N}(96)$ & $2.615(5)$ \\
\hline $\mathrm{Bi}(2)-\mathrm{N}(91)$ & $2.649(5)$ \\
\hline $\mathrm{Bi}(2)-\mathrm{C}(77)$ & $2.816(10)$ \\
\hline
\end{tabular}

Bond angles (deg.)

$\begin{array}{ll}\mathrm{C}(11)-\mathrm{Bi}(1)-\mathrm{O}(31) & 82.32(14) \\ \mathrm{C}(11)-\mathrm{Bi}(1)-\mathrm{N}(46) & 84.89(12) \\ \mathrm{O}(31)-\mathrm{Bi}(1)-\mathrm{N}(46) & 82.61(12) \\ \mathrm{C}(11)-\mathrm{Bi}(1)-\mathrm{O}(22) & 86.7(3) \\ \mathrm{O}(31)-\mathrm{Bi}(1)-\mathrm{O}(22) & 86.8(2) \\ \mathrm{N}(46)-\mathrm{Bi}(1)-\mathrm{O}(22) & 167.2(3) \\ \mathrm{C}(11)-\mathrm{Bi}(1)-\mathrm{O}(21) & 89.0(3) \\ \mathrm{O}(31)-\mathrm{Bi}(1)-\mathrm{O}(21) & 138.2(2) \\ \mathrm{N}(46)-\mathrm{Bi}(1)-\mathrm{O}(21) & 137.4(2) \\ \mathrm{O}(22)-\mathrm{Bi}(1)-\mathrm{O}(21) & 51.9(3) \\ \mathrm{C}(11)-\mathrm{Bi}(1)-\mathrm{N}(41) & 85.64(13) \\ \mathrm{O}(31)-\mathrm{Bi}(1)-\mathrm{N}(41) & 146.04(12) \\ \mathrm{N}(46)-\mathrm{Bi}(1)-\mathrm{N}(41) & 64.72(10) \\ \mathrm{O}(22)-\mathrm{Bi}(1)-\mathrm{N}(41) & 124.2(2) \\ \mathrm{O}(21)-\mathrm{Bi}(1)-\mathrm{N}(41) & 72.8(2) \\ \mathrm{C}(61)-\mathrm{Bi}(2)-\mathrm{O}(81) & 83.93(14) \\ \mathrm{C}(61)-\mathrm{Bi}(2)-\mathrm{O}(72) & 85.6(4) \\ \mathrm{O}(81)-\mathrm{Bi}(2)-\mathrm{O}(72) & 77.0(3) \\ \mathrm{C}(61)-\mathrm{Bi}(2)-\mathrm{O}(71) & 86.2(5) \\ \mathrm{O}(81)-\mathrm{Bi}(2)-\mathrm{O}(71) & 129.5(4) \\ \mathrm{O}(72)-\mathrm{Bi}(2)-\mathrm{O}(71) & 52.9(4) \\ \mathrm{C}(61)-\mathrm{Bi}(2)-\mathrm{N}(96) & 83.27(18) \\ \mathrm{O}(81)-\mathrm{Bi}(2)-\mathrm{N}(96) & 87.7(2) \\ \mathrm{O}(72)-\mathrm{Bi}(2)-\mathrm{N}(96) & 161.9(4) \\ \mathrm{O}(71)-\mathrm{Bi}(2)-\mathrm{N}(96) & 139.9(3) \\ \mathrm{C}(61)-\mathrm{Bi}(2)-\mathrm{N}(91) & 82.49(18) \\ \mathrm{O}(81)-\mathrm{Bi}(2)-\mathrm{N}(91) & 148.2(2) \\ \mathrm{O}(72)-\mathrm{Bi}(2)-\mathrm{N}(91) & 130.1(3) \\ \mathrm{O}(71)-\mathrm{Bi}(2)-\mathrm{N}(91) & 78.0(3) \\ \mathrm{N}(96)-\mathrm{Bi}(2)-\mathrm{N}(91) & 62.32(19) \\ & \\ & \\ & \end{array}$




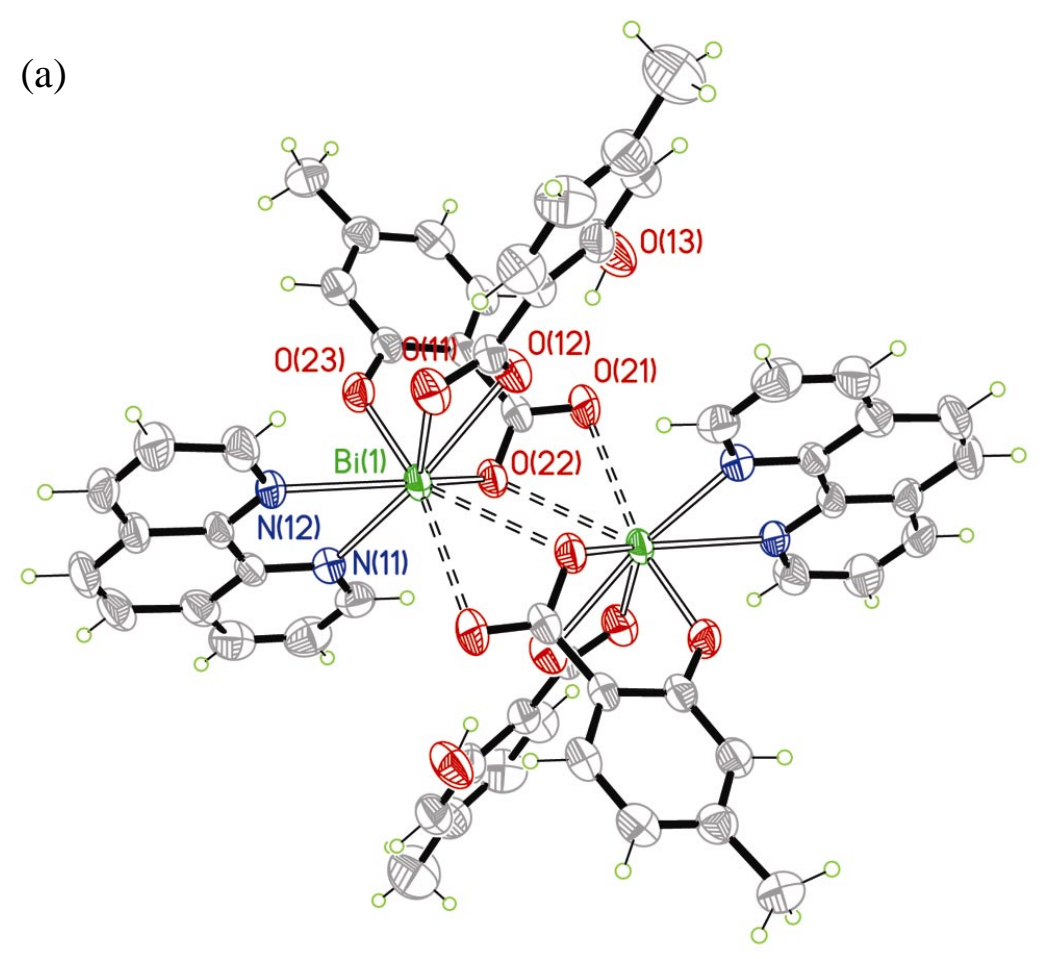

(b)

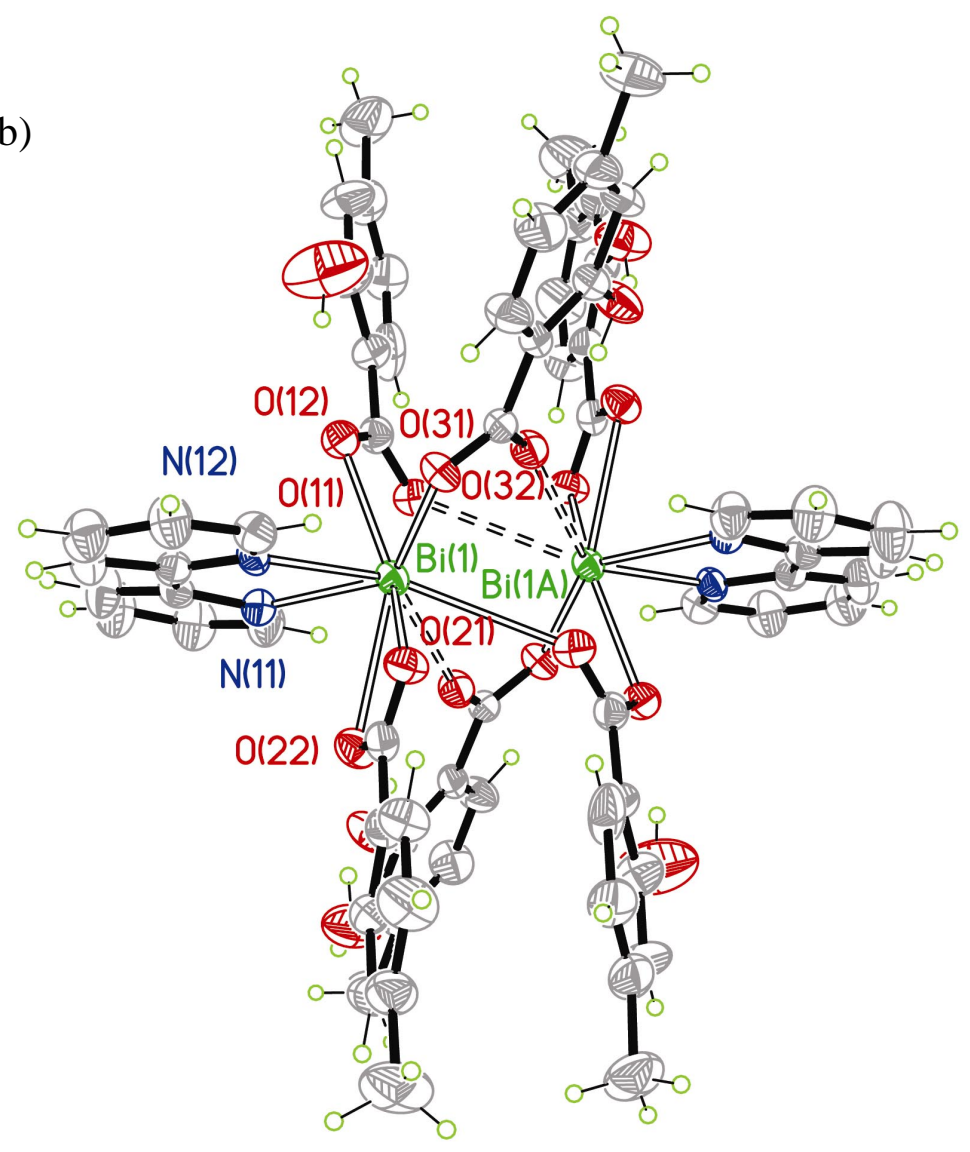

Figure S6. Coordination environment of $\mathrm{Bi}(\mathrm{III})$ in (a) $\left[\mathbf{9} \cdot \mathrm{Me}_{2} \mathrm{CO}\right]_{2}$ and (b) $\left[\mathbf{1 0} \cdot 2 \mathrm{Me}_{2} \mathrm{CO}\right]_{2}$. 


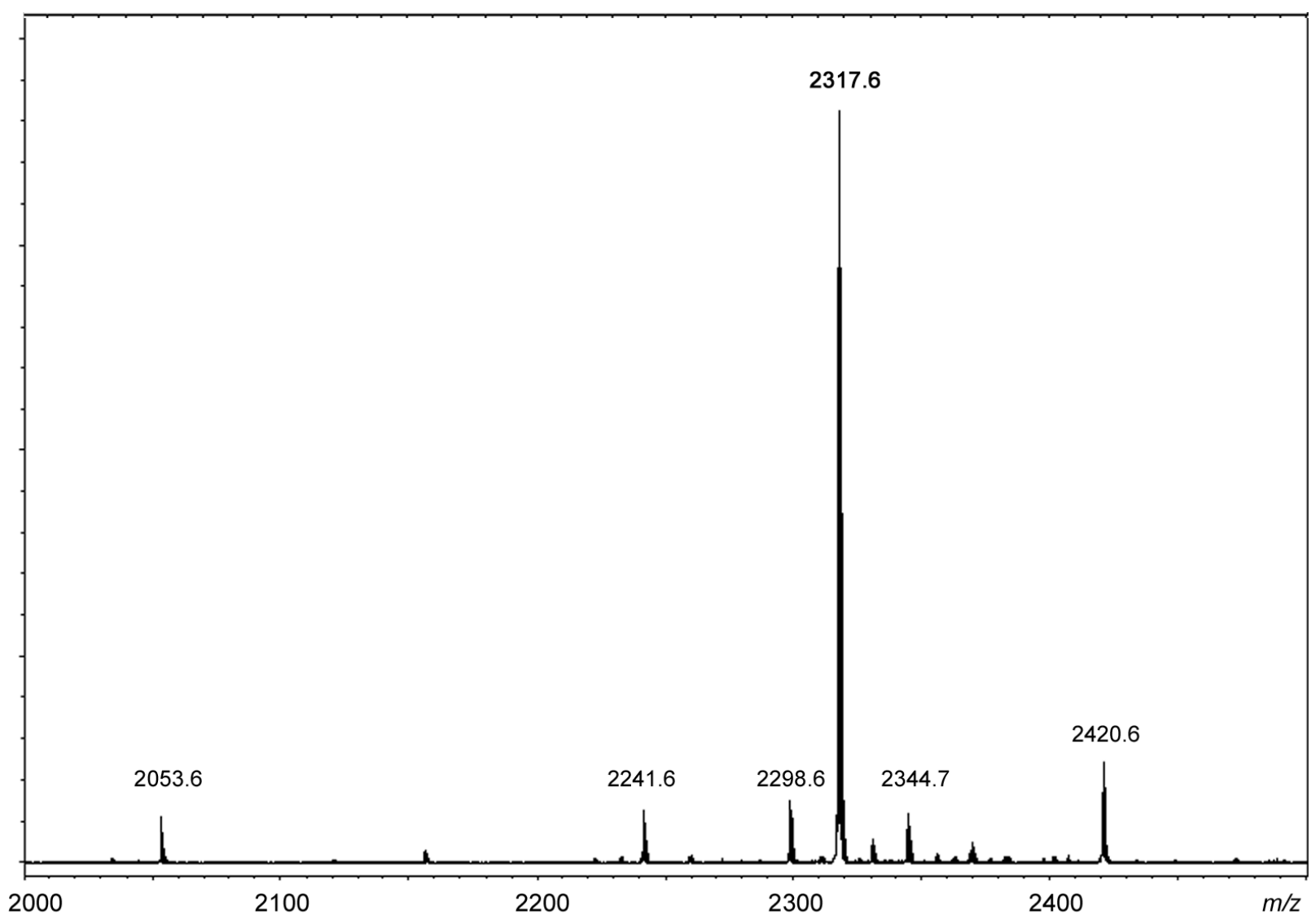

Figure S7. The negative-ion ESI mass spectrum of $\mathbf{1 2}$ run in an acetone mobile phase. 

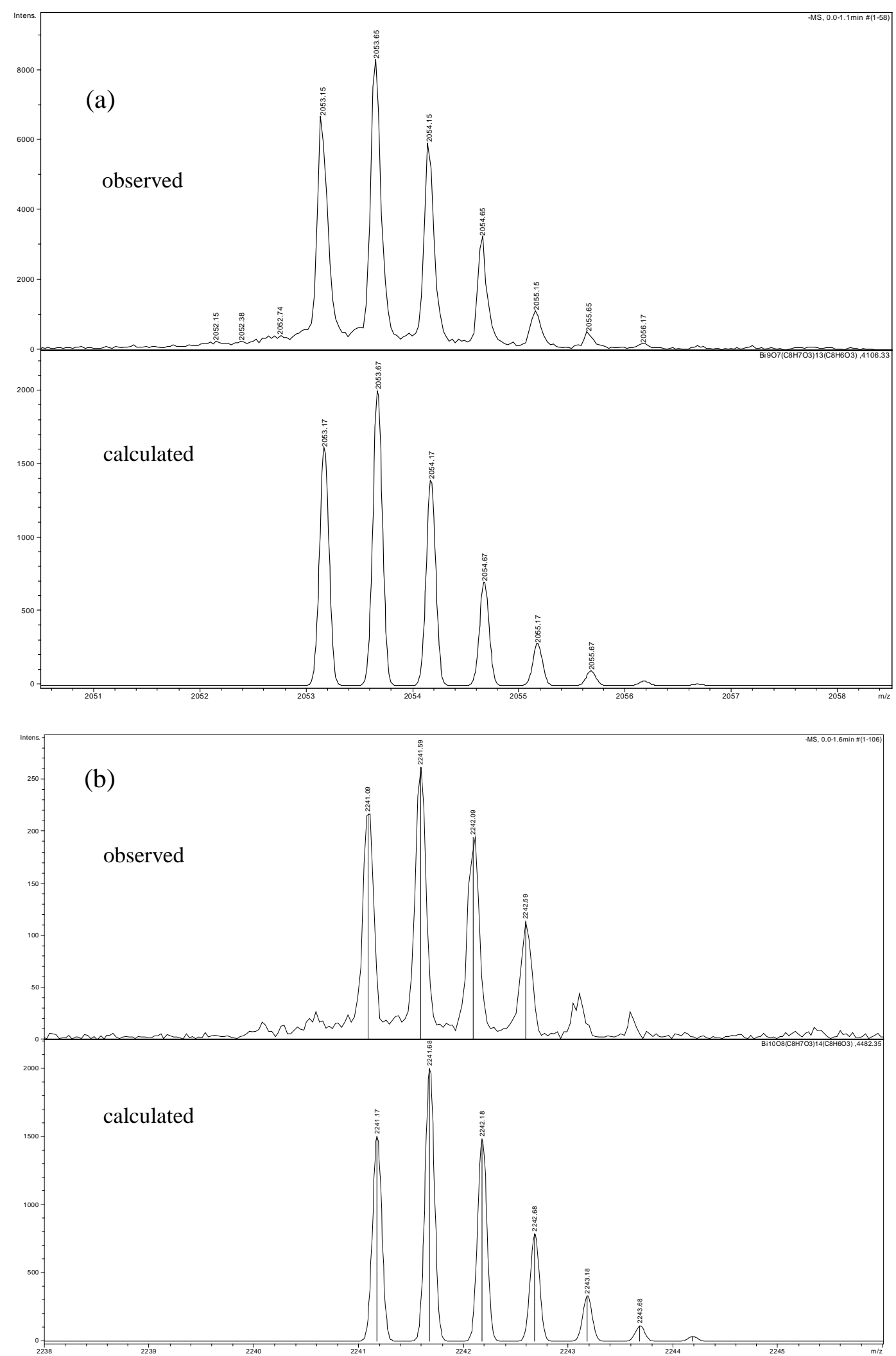

Figure S8. Comparison of experimental and calculated negative-ion ESI mass spectral isotope patterns for $\left[\mathrm{Bi}_{9} \mathrm{O}_{7}\left(\mathrm{Hsal}^{4 \mathrm{Me}}\right)_{13}(\mathrm{sal})\right]^{2-}(\mathbf{a})$ and $\left[\mathrm{Bi}_{10} \mathrm{O}_{8}\left(\mathrm{Hsal}^{4 \mathrm{Me}}\right)_{14}\left(\mathrm{sal}^{4 \mathrm{Me}}\right)\right]^{2-}(\mathbf{b})$. 

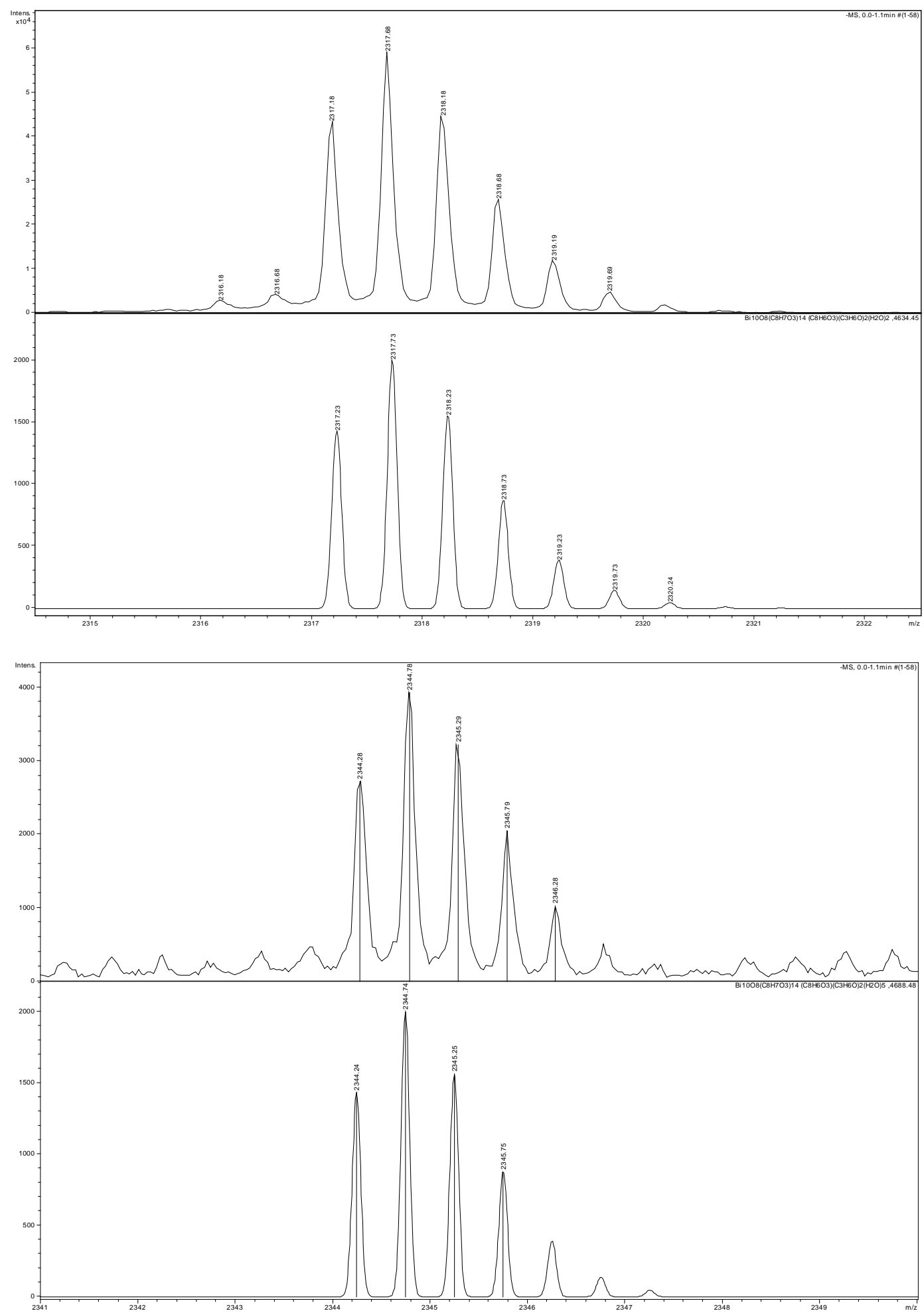

Figure S9. Comparison of experimental and calculated negative-ion ESI mass spectral isotope patterns for $\left[\mathrm{Bi}_{10} \mathrm{O}_{8}\left(\mathrm{Hsal}^{4 \mathrm{Me}}\right)_{14}\left(\mathrm{sal}^{4 \mathrm{Me}}\right)\left(\mathrm{Me}_{2} \mathrm{CO}\right)_{2}\left(\mathrm{H}_{2} \mathrm{O}\right)_{2}\right]^{2-}(\mathbf{a})$ and $\left[\mathrm{Bi}_{10} \mathrm{O}_{8}\left(\mathrm{Hsal}^{4 \mathrm{Me}}\right)_{14}\left(\mathrm{sal}^{4 \mathrm{Me}}\right)\left(\mathrm{Me}_{2} \mathrm{CO}\right)_{2}\left(\mathrm{H}_{2} \mathrm{O}\right)_{5}\right]^{2-}(\mathbf{b})$. 